\section{SP0190 2019 EULAR RECOMMENDATIONS FOR THE MANAGEMENT OF SJÖGREN'S SYNDROME WITH TOPICAL AND SYSTEMIC THERAPIES}

Manuel Ramos-Casals ${ }^{1} .{ }^{1}$ Hospital Clinic, Autoimmune Diseases, Barcelona, Spain Manuel Ramos-Casals ${ }^{1,2,3}$, Pilar Brito-Zerón ${ }^{2,4}$, Stefano Bombardieri ${ }^{5}$, Hendrika Bootsma ${ }^{6}$, Salvatore De Vita ${ }^{7}$, Thomas Dörner ${ }^{8}$, Benjamin A. Fisher ${ }^{9}$, Jacques-Eric Gottenberg ${ }^{10}$, Gabriela Hernández-Molina ${ }^{11}$, Agnes Kocher ${ }^{12}$, Belchin Kostov ${ }^{13}$, Aike A. Kruize ${ }^{14}$, Thomas Mandl ${ }^{15}$, Wan-Fai Ng ${ }^{16}$ Soledad Retamozo ${ }^{17}$, Raphaèle Seror ${ }^{18}$, Yehuda Shoenfeld ${ }^{19}$, Antoni SisóAlmirall $^{13,20}$, Athanasios G. Tzioufas ${ }^{21}$, Claudio Vitali ${ }^{22}$, Simon Bowman ${ }^{9}$, Xavier Mariette ${ }^{18}$, on behalf of the EULAR-Sjögren Syndrome Task Force Group. ${ }^{1}$ Department of Autoimmune Diseases, ICMiD, Hospital Clínic, Barcelona, Spain; ${ }^{2}$ Laboratory of Autoimmune Diseases Josep Font, IDIBAPS-CELLEX, Barcelona, Spain; ${ }^{3}$ University of Barcelona, Hospital Clínic, Barcelona, Spain; ${ }^{4}$ Autoimmune Diseases Unit, Department of Medicine, Hospital CIMA- Sanitas, Barcelona, Spain; ${ }^{5}$ Rheumatology Unit, University of Pisa, Pisa, Italy, ${ }^{6}$ Department of Rheumatology and Clinical Immunology, University of Groningen, University Medical Center Groningen, Groningen, The Netherlands; ${ }^{7}$ Clinic of Rheumatology, Department of Medical and Biological Sciences, University Hospital "Santa Maria della Misericordia", Udine, Italy; ${ }^{8} \mathrm{Karl}$ Landsteiner Institute for Physical Medicine and Rehabilitation, and Department of Physical Medicine and Rehabilitation, Kaiser Franz Josef-Hospital, SMZ Süd, Vienna, Austria; ${ }^{9}$ Rheumatology Department, University Hospitals Birmingham NHS Foundation Trust, National Institute for Health Research, Birmingham Biomedical Research Centre, Birmingham, United Kingdom; ${ }^{10}$ Department of Rheumatology, Strasbourg University Hospital, Université de Strasbourg, CNRS, Strasbourg, France; ${ }^{11}$ Immunology and Rheumatology Department, Instituto Nacional de Ciencias Médicas y Nutrición Salvador Zubirán. México City, Mexico; ${ }^{12}$ Department of Rheumatology, Immunology and Allergology, Inselspital University Hospital Bern, Switzerland and Institute of Nursing Science (INS), Department Public Health (DPH), Faculty of Medicine, University of Basel, Switzerland; ${ }^{13}$ Primary Healthcare Transversal Research Group, IDIBAPS Barcelona, Spain; ${ }^{14}$ Department of Rheumatology and Clinical Immunology, University Medical Center Utrecht, Utrecht, The Netherlands ${ }^{15}$ Department of Rheumatology, Skane University Hospital Malmö, Lund University, Lund, Sweden; ${ }^{16}$ Institute of Cellular Medicine, Newcastle University, Newcastle Upon Tyne, United Kingdom; ${ }^{17}$ Instituto Modelo de Cardiología Privado S.R.L., Córdoba, Argentina, IUCBC, INICSA, CONICET, Córdoba, Argentina; ${ }^{18}$ Université Paris-Sud; Assistance Publique-Hôpitaux de Paris, Hôpitaux Universitaires Paris-Sud, Department of Rheumatology, INSERM U1184, Le Kremlin Bicêtre, France; ${ }^{19}$ Zabludowicz Center for Autoimmune Diseases, Sheba Medical Center, Tel Aviv University, 5265601, Tel Hashomer, Israel; ${ }^{20}$ Centre d'Assistència Primària ABS Les Corts, CAPSBE, Barcelona, Spain; ${ }^{21}$ Department of Pathophysiology, School of Medicine, University of Athens, Athens, Greece; ${ }^{22}$ Villa San Giuseppe, Istituto S. Stefano, Como, Italy

Aims: The therapeutic management of Sjögren syndrome (SjS) has not changed substantially over the past decades: treatment decisions remain challenging in clinical practice, without a specific therapeutic target beyond the relief of symptoms as the most important goal. In view of this scenario, the European League Against Rheumatism (EULAR) promoted and supported an international collaborative study (EULAR SS Task Force) aimed to develop the first EULAR evidence-, consensus-based recommendations for the management of patients with SjS with topical and systemic medications. The aim was to develop a rational therapeutic approach to SjS patients useful for healthcare professionals, doctors in specialist training, medical students, pharmaceutical industries and drug regulatory organizations following the 2014 EULAR standardized operating procedures.

Methods: The Task Force included rheumatologists, specialists in internal medicine, oral care specialists, ophthalmologists, gynaecologists, dermatologists, epidemiologists, statisticians, GPs, nurses and patient representatives from 30 countries of the 5 continents. Evidence was collected from studies including primary SjS patients fulfilling the 2002/2016 criteria; when no evidence was available, evidence from studies including associated SjS or patients fulfilling previous sets of criteria was considered and extrapolated. On the basis of the research questions, a systematic literature search between January 1986 and December 2017 was carried out. Summary-of-findings tables were generated and levels of evidence were determined according to the study design using the standards of the Oxford CEBM. A web-based Delphi procedure was carried out in order to reach consensus. For each statement, the grade of recommendation was based on the maximum level of evidence achieved in the SLR using the 4-point scale CEBM categorization. Consensus for endorsement was defined as an agreement score of at least 8 on a $0-10$ scale by more than $80 \%$ of participants.

Results: The Task Force endorsed the presentation of general principles for the management of patients with SjS as 3 overarching, general consensus-based recommendations and 12 specific recommendations that form a logical sequence starting with the management of the central triplet of symptoms (dryness, fatigue and pain) followed by the management of systemic, extraglandular disease (Table 1).

Conclusion: The 2019 EULAR recommendations are based on the evidence collected in the last 16 years in the area of management of primary 2002 SjS patients and on discussions by a large and broadly international Task Force. The recommendations synthesise the current thinking on approaching SjS treatment in a set of overarching principles and recommendations. We hope that the current recom mendations will be broadly applied in clinical practice and/or serve as a template for national societies to develop local recommendations.

Abstract SP0190 - Table 1. Overarching and specific recommendations.

\begin{tabular}{|c|c|c|c|}
\hline & $\begin{array}{c}\text { LoA ( } \% \\
\text { endorsement) }\end{array}$ & LoE & GoR \\
\hline $\begin{array}{l}\text { A. Patients with SjS should be managed at, or in close } \\
\text { collaboration with, centres of expertise following a } \\
\text { multidisciplinary approach }\end{array}$ & 90,7 & na & $\mathrm{Na}$ \\
\hline $\begin{array}{l}\text { B. The first therapeutic approach for dryness should be } \\
\text { symptomatic relief using topical therapies }\end{array}$ & 93,3 & na & $\mathrm{Na}$ \\
\hline $\begin{array}{l}\text { C. Systemic therapies may be considered for treating } \\
\text { active systemic disease }\end{array}$ & 90,7 & na & $\mathrm{Na}$ \\
\hline $\begin{array}{l}\text { 1. Baseline evaluation of salivary gland function is } \\
\text { recommended before starting treatment for oral } \\
\text { dryness }\end{array}$ & 81,3 & 5 & $D$ \\
\hline $\begin{array}{l}\text { 2. The preferred first therapeutic approach for oral } \\
\text { dryness according to salivary gland function may be: } \\
\text { 2.1. Non-pharmacological stimulation for mild dysfunction; } \\
\text { 2.2. Pharmacological stimulation* for moderate dysfunction; } \\
\text { 2.3. Saliva substitution for severe dysfunction }\end{array}$ & 88,0 & $\begin{array}{l}1 \mathrm{a} / \\
{ }^{*} 1 \mathrm{~b}\end{array}$ & B \\
\hline $\begin{array}{l}\text { 3. First-line therapeutic approach of ocular dryness } \\
\text { includes the use of artificial tears and ocular gels/ } \\
\text { ointments }\end{array}$ & 98,7 & $1 a$ & B \\
\hline $\begin{array}{l}\text { 4. Refractory/severe ocular dryness may be managed } \\
\text { using topical immunosuppressive-containing drops }{ }^{\star} \\
\text { and autologous serum eye drops }\end{array}$ & 94,7 & $\begin{array}{l}1 \mathrm{a} / \\
{ }^{*} 1 \mathrm{~b}\end{array}$ & $\mathrm{~B} / \mathrm{D}$ \\
\hline $\begin{array}{l}\text { 5. Concomitant diseases should be evaluated in } \\
\text { patients presenting with fatigue/pain, whose severity } \\
\text { should be scored using specific tools }\end{array}$ & 93,3 & 5 & $\mathrm{D}$ \\
\hline $\begin{array}{l}\text { 6. Consider analgesics or other pain-modifying agents } \\
\text { for musculoskeletal pain considering the balance } \\
\text { between potential benefits and side-effects }\end{array}$ & 89,3 & 4 & $C$ \\
\hline $\begin{array}{l}\text { 7. Treatment of systemic disease should be tailored to } \\
\text { organ-specific severity using the ESSDAI definitions }\end{array}$ & 89,3 & 4 & C \\
\hline $\begin{array}{l}\text { 8. Glucocorticoids should be used at the minimum } \\
\text { dose and length of time necessary to control active } \\
\text { systemic disease }\end{array}$ & 85,3 & 4 & $C$ \\
\hline $\begin{array}{l}\text { 9. Immunosuppressive agents should be mainly used } \\
\text { as GC-sparing agents, with no evidence supporting the } \\
\text { choice of one agent over the others }\end{array}$ & 82,7 & 4 & $C$ \\
\hline $\begin{array}{l}\text { 10. B-cell targeted therapies may be considered in } \\
\text { patients with severe, refractory systemic disease }\end{array}$ & 98,7 & $1 b$ & $D$ \\
\hline $\begin{array}{l}\text { 11. Systemic organ-specific therapeutic approach may } \\
\text { follow as a general rule the sequential (or combined) use of } \\
\text { glucocorticoids, immunosuppressive agents and biologics }\end{array}$ & 98,7 & 5 & $D$ \\
\hline $\begin{array}{l}\text { 12. Treatment of B-cell lymphoma should be } \\
\text { individualized according to the specific histological } \\
\text { subtype and disease stage }\end{array}$ & 88,0 & 4 & C \\
\hline
\end{tabular}

Disclosure of Interests: None declared

DOI: 10.1136/annrheumdis-2019-eular.8570

\section{SP0191 MANAGEMENT OF ADULT APS: RECOMMENDATIONS FROM AN EULAR TASK FORCE}

Maria Tektonidou. National and Kapodistrian University of Athens, Joint Rheumatology Program, Greece

Background: Antiphospholipid syndrome (APS) is a systemic autoimmune disorder characterized by arterial and venous thrombotic events or pregnancy morbidity in the presence of persistently positive antiphospholipid antibodies. There is a great heterogeneity in clinical and laboratory classification of the syndrome and treatement approaches over the past four decades.

Objectives: To develop evidence-based recommendations for the prevention and management of adult APS that will help guide clinical practice.

Methods: EULAR standardised operating procedures were followed. A task force was formed including 21 physicians, 1 healthcare professional, and 2 patients, from 11 countries. Research questions were developed using the Delphi method. A systematic literature review of published articles up to January 2018 was performed. Results: Based on the evidence and experts opinion, a set of 3 overarching principles and 12 recommendations including their level of evidence and grade of recommendation was developed and voted. The level of agreement for each recommendation was high. The overarching principles include the risk stratification, general measures, and education/counselling of patients with APS.

Recommendations for primary prevention address the primary thromboprophylaxis (low dose aspirin vs no treatment) in antiphospholipid antibody individuals 
including asymptomatic carriers, patients with systemic lupus erythematosus without history of thrombotic or obstetric APS, and non-pregnant women with a history of obstetric APS only. Recommendations for the management of thrombotic manifestations address different types of anticoagulation in patients with definite APS and first provoked or unprovoked venous thrombosis and the management of recurrent venous thrombosis, as well as the type and intensity of anticoagulation in patients with first or recurrent arterial thrombosis.

Recommendations for the management of obstetric APS describe the management of various types of pregnancy complications in APS and of refractory to treatment cases. Recommendations for catastrophic APS refer to precipitating factors, first-line treatment of catastrophic APS, and management of refractory cases. Conclusion: These recommendations based on evidence and expert opinion aim to guide practice and improve quality of care in patients with APS.

\section{REFERENCE:}

[1] Tektonidou MG, Andreoli L, Limper M, et al. EULAR Recommendations for the management of Antiphospholipid Syndrome in adults. Ann Rheum Dis. 2019

Disclosure of Interests: None declared

DOI: 10.1136/annrheumdis-2019-eular.8601

\section{SP0192 UPDATE OF THE EULAR RECOMMENDATIONS LAGE VESSEL VASCULITIS MANAGEMENT}

Bernhard Hellmich. Medius Kliniken, Klinik für Innere Medizin, Rheumatologie und Immunologie, Kirchheim-Teck, Germany

Background: Since the publication of the European League Against Rheumatism (EULAR) recommendations for the management of large vessel vasculitis (LVV) in 2009, several relevant randomized clinical trials and cohort analyses have been published, which have the potential to change clinical care and therefore supporting the need to update the original recommendations.

Objectives: To update the 2009 EULAR recommendations for the management of LVV.

Methods: Using EULAR standardized operating procedures for EULARendorsed recommendations, the EULAR task force undertook a systematic literature review and sought opinion from 20 experts from 13 countries. We modified existing recommendations and created new recommendations.

Results: Three overarching principles and 10 recommendations were formulated. We recommend that a suspected diagnosis of LVV should be confirmed by imaging or histology. High dose glucocorticoid therapy (40-60 mg/day prednisone-equivalent) should be initiated immediately for induction of remission in active giant cell arteritis (GCA) or Takayasu arteritis (TAK). We recommend adjunctive therapy in selected patients with GCA (refractory or relapsing disease, presence of an increased risk for glucocorticoid-related adverse events or complications) using tocilizumab. Methotrexate may be used as an alternative. Non-biologic glucocorticoid-sparing agents should be given in combination with glucocorticoids in all patients with TAK and biologic agents may be used in refractory or relapsing patients. We no longer recommend the routine use of antiplatelet or anticoagulant therapy for treatment of LVV unless it is indicated for other reasons.

Conclusion: We have updated the recommendations for the management of LVV to facilitate the translation of current scientific evidence and expert opinion into better management and improved outcome of patients in clinical practice. Disclosure of Interests: Bernhard Hellmich Consultant for: Roche, Speakers bureau: Abbvie, MSD, Roche, Novartis, Pfizer

DOI: 10.1136/annrheumdis-2019-eular.8496

SATURDAY, 15 JUNE 2019

12:00:00 - 13:30:00

\section{Skin and eye manifestations in rheumatic diseases_}

\section{SP0193 WIN: SKIN AND RHEUMATIC DISEASES}

Annegret Kuhn. University Hospital Muenster, Executive Department of the University Hospital, Muenster, Germany

Background: The main underlying mechanisms of skin manifestations in rheumatic diseases include autoimmune responses, auto-inflammatory processes, and tissue-specific alterations. In psoriasis, therapies targeting $T$ cell activation, $T$ cell migration, or neutralization of disease-related cytokines are highly effective. However, targeted therapies still need to be developed in less frequent autoimmune diseases, such as systemic lupus erythematosus (SLE), systemic sclerosis (SSc), or dematomyositis (DM).

Objectives: To provide an update for rheumatologists on the management of skin manifestations in autoimmune diseaes based on recent insights into the pathogenesis.
Methods: Several agents are approved for the treatment of SLE, including the monoclonal antibody belimumab, a B lymphozyte stimulator-specific inhibitor, but no drugs have been licensed specifically for the treatment of skin manifestations in this disease. The aim of the European guideline was to achieve a broad consensus on treatment strategies for patients with cutaneous lupus erythematosus (CLE) by a European subcommittee. In total, 16 European participants were included in this project and agreed on all recommendations.

Results: First-line treatment options in CLE include topical corticosteroids or calcineurin inhibitors; in patients with disfiguring and widespread disease, systemic agents need to be applied. The first-line systemic treatment is antimalarials, but some patients are therapy-resistant and immunosuppressive agents, such as methotrexate, are used as alternative therapeutic option. In 2011, the monoclonal antibody belimumab was introduced for SLE as an adjunct therapy for patients with autoantibody-positive disease who despite standard therapy show high disease activity, intolerance of other treatments, or an unacceptably high need for corticosteroids. So far, a validated skin score has not been used to confirm the efficacy of belimumab on mucocutaneous manifestations. In SSc, the therapeutic modalities are even more limited. Treatment with endothelin-receptor antagonists has been proven to reduce the occurrence of new digital ulcers in SSc patients but has no or limited effect on healing of digital ulcers. DM is a further idiopathic autoimmune disease characterized by inflammation of the muscles and skin which is treated with immunosuppressives. Corticosteroids are still the first-line treatment for muscle involvement in DM, but skin lesions often flare by reduction or discontinuation.

Conclusion: In summary, there is a high unmet need for new therapeutic strategies focusing on skin involvement in systemic autoimmune diseases. Therefore innovative designs of randomized controlled trials based on the pathogenesis are warranted to develop new therapies for patients with skin manifestations in rheumatic diseases.

\section{REFERENCES:}

[1] Kuhn A, Landmann A, Bonsmann G. The skin in autoimmune diseases unmet needs. Autoimmun Rev 2016;15:948-954

[2] Kuhn A, Aberer E, Bata-Csoergő Z, et al. S2k guideline for treatment of cutaneous lupus erythematosus - guided by the European Dermatology Forum (EDF) in cooperation with the European Academy of Dermatology and Venereology (EADV). J Eur Acad Dermatol Venereol 2017;31:389-404

[3] Smith V, Scirè CA, Talarico R, et al. Systemic sclerosis: state of the art on clinical practice guidelines. RMD Open 2018;4:e000782

[4] Sunderkoetter C, Nast A, Worm M, et al. Guidelines on dermatomyositis excerpt from the interdisciplinary $\mathrm{S} 2 \mathrm{k}$ guidelines on myositis syndromes by the German Society of Neurology. J Dtsch Dermatol Ges 2016;14:321-338

[5] Tamirou F, Arnaud L, Talarico R, et al. Systemic lupus erythematosus: state of the art on clinical practice guidelines. RMD Open 2018;4:e000793

Disclosure of Interests: Annegret Kuhn Grant/research support from: Biogen, Galderma, GlaxoSmithKline, LeoPharma, Speakers bureau: La Roche Posay DOI: 10.1136/annrheumdis-2019-eular.8522

SATURDAY, 15 JUNE 2019

12:00:00 - 13:30:00

\section{To image or not to image in spondyloarthritis?}

\section{SP0194 WHEN AND HOW TO USE AND NOT USE IMAGING FOR DIAGNOSIS?}

Floris A. van Gaalen. Leiden University Medical Center, Rheumatology, Leiden, Netherlands

Since axial spondyloarthritis (axSpA) has no single shared distinguishing feature that distinguishes the disease from other causes of back pain, diagnosis of axSpA requires recognition by the clinician of a pattern of features that taken together are characteristic of axSpA. Information from patient history and physical examination, laboratory, and imaging findings may all aid in recognition of axSpA and the diagnosis of axSpA requires exclusion of other potential causes for these abnormalities/differences.

Traditionally, plain radiography is used to assess sacroiliac and spinal involvement of disease. A weakness of radiography in assessing sacroiliac involvement apart from its inability to detect early disease - is reader variability. Additionally, involvement of the spine (i.e detection of syndesmophytes) is very uncommon in early disease.

The use of MRI in diagnosing axSpA has increased over the past ten years and $\mathrm{MRI}$ greatly facilitates earlier diagnosis of axSpA. However, it has also become increasingly clear that MRI detectable lesions associated with SpA -including bone marrow edema and fatty lesions- may also occur in patients without axSpA. 